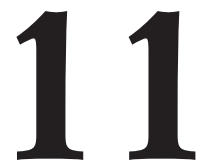

\title{
UNA APROXIMACIÓN A LOS PATRONES DE COMUNICACIÓN ENTRE EL PROFESOR MENTOR Y EL PROFESOR-ESTUDIANTE EN EL CONTEXTO DE LA PRÁCTICA PEDAGóGICA
}

\author{
(AN APPROACH TO COMMUNICATION PATTERNS BETWEEN \\ TEACHERS AND STUDENT-TEACHERS IN THE CONTEXT OF \\ PEDAGOGICAL PRACTICE)
}

Claudio Díaz Larenas

Universidad de Concepción (Chile)

Cecilia Bastías Díaz

Universidad San Sebastián (Concepción, Chile)

\section{RESUMEN}

Puesto que la Formación Inicial Docente (FID) ha sido, en este último tiempo, parte de la agenda nacional del Ministerio de Educación de Chile, este estudio tiene como objetivo comprender los patrones de comunicación establecidos entre un grupo de profesores mentores y de profesores-estudiantes de pedagogía de una institución de educación superior de Concepción, Chile.

La investigación es un estudio de casos múltiples de doce informantes, adscrita al paradigma interpretativo-hermeneútico. El instrumento de recolección de información es la entrevista semiestructurada, que fue analizada mediante el uso del software de análisis cualitativo Atlas-Ti que permitió segmentar, codificar y establecer relaciones entre los datos.

El estudio constató que los patrones de comunicación en un proceso de mentoría se caracterizan por establecer tipos de interacciones reflexivas y promover la co-construcción de significados y la permanente autocrítica del quehacer pedagógico. Asimismo, el rol del mentor como apoyo y guía, resulta crucial en su relación con el profesor-estudiante.

\section{ABSTRACT}

Since initial teacher training has been taken into account in the national agenda of the Ministry of Education in Chile, this research aims at understanding the patterns of communication established between a group of mentors and 
a group of student-teachers in a Pedagogy programme in a university located in Concepción (Chile).

The research is a hermeneutic-analytic multiple case study. The research instrument was a semi-structured interview that was analysed through the use of the qualitative software Atlas-Ti that let the segmentation, codification and relationship explorations of the data.

The research findings reveal that the participants' patterns of communication are featured by reflective interactions which enhance the co-construction of meanings and ongoing self-reflection upon their classroom teaching practices. In addition, the supportive, counselling and helpful roles of the mentor, are key components.

\section{INTRODUCCIÓN}

A partir del año 1998 el fortalecimiento de la Formación Inicial Docente (FID) ha sido uno de los temas de la agenda nacional del Ministerio de Educación -MINEDUC_ de Chile (Ávalos, 2002), con el propósito de mejorar las prácticas pedagógicas de los profesores en formación y, a la vez, responder a los requerimientos de las políticas curriculares del país.

La institución superior en la que se desarrolla esta investigación cuenta con un programa de prácticas pedagógicas tempranas. No obstante, éste presenta una serie de dificultades, a saber: desvinculación entre la universidad y los centros de práctica, mayor número de estudiantes de práctica en relación con la cantidad de supervisores, inexistencia de un equipo interdisciplinario con dedicación exclusiva al área de práctica e inexistencia de un mismo lenguaje entre la universidad y el establecimiento educacional respectivo.

A la luz de estas consideraciones, Nation y Macalister (2010) señalan que la formación inicial de profesores de inglés requiere de un fuerte componente de prácticas pedagógicas a través de la formación de mentores en las escuelas, que colaboren con la formación de los estudiantes y sintonicen en el mismo lenguaje de la institución universitaria. Cabe señalar que a partir de los años ochenta y noventa, la implementación e institucionalización de programas de mentoría ha sido muy valorada en el ámbito educacional en países, tales como el Reino Unido, Estados Unidos, Hungría, Rumania, Israel y Australia. Estos programas responden a la necesidad de: bajar los niveles de deserción de la carrera docente, mejorar las prácticas pedagógicas y promover el desarrollo profesional de los profesores en formación, noveles, o experimentados a través del apoyo y asistencia profesional in-situ de un mentor (Feiman-Nemser, 2003; Prieto, 2008; Pimienta, 2008). 
En el contexto nacional del mejoramiento de las prácticas pedagógicas en Chile, entre los años 2004-2005 se implementó el programa piloto de entrenamiento de mentores para profesores de inglés de establecimientos educacionales públicos y semi-públicos, impulsado por el programa «Inglés Abre Puertas» (PIAP) del MINEDUC (http://www.ingles.mineduc.cl/). Este proceso culminó con la formación de cien mentores en el país, de los cuales siete están vinculados a la institución superior donde se realiza este estudio, y han implementado procesos de mentoría en el área de prácticas pedagógicas de la carrera de Pedagogía en Inglés.

La contribución principal de esta investigación se enmarca en la comprensión de los patrones de comunicación que se establecen en la relación de mentoría dentro de un contexto chileno y el impacto que estos procesos pueden tener en los programas de formación docente.

\section{MARCO TEÓRICO}

\subsection{Definición y características de la mentoría}

Las conceptualizaciones dadas por diferentes autores responden a un enfoque socio-cognitivo y socio-constructivista pues las interacciones que se establecen entre un profesor mentor y un profesor estudiante contribuyen al aprendizaje mutuo, la intersubjetividad y la co-construcción de significados (Feiman-Nemser, 2003; Orland-Barak, 2005; Sotomayor y Walker, 2009). Asimismo, la mentoría se concibe como una relación dinámica e interactiva de intercambio profesional entre individuos con diferentes niveles de experiencia y conocimiento, siendo función del más experimentado - mentorguiar y asistir al profesional novel o en formación - profesor-estudiantecon el propósito de promover el desarrollo profesional de este último. No obstante, este proceso requiere del mentor, cualidades de liderazgo, conocimiento y destrezas personales que permitan desarrollar un modelo de crecimiento mutuo. El rol del mentor es ser un interlocutor activo, una «caja de resonancia», de ideas y «reflejo» del accionar del profesor-estudiante, para avanzar en el continuo desarrollo profesional y personal.

\subsection{Roles y funciones del mentor}

$\mathrm{Al}$ revisar la literatura relacionada con programas de mentoría institucionalizados en países como: Estados Unidos, Reino Unido e Israel la definición de los roles y funciones del mentor — desde la visión academicista- concuerda con una orientación centrada en el desarrollo personal y profesional del profesor-estudiante, novicio o en ejercicio, en la que el 
mentor cumple roles de aculturador, apoyo, guía, educador, facilitador, evaluador y consejero (Feiman-Nemser, 2003; Malderez y Wedell, 2007; Randall y Thornton, 2001).

Según Orland-Barak (2005) las funciones del mentor son: guiar y apoyar la reflexión, explorar y construir el pensamiento del profesor-estudiante; facilitar la inserción en el establecimiento educacional; ser agente de cambio; incentivar modelos pedagógicos innovadores; ser fuente de conocimiento; identificar nudos críticos en el desempeño docente; promover un plan de acción y hacer seguimiento de los mismos; y evaluar el desempeño docente al final del proceso de acompañamiento.

\subsection{Tipos de intervención al interior del proceso de mentoría}

Orland-Barak (2003) plantea tres estilos de intervención, a saber: directivas, alternativas, y no directivas. Las intervenciones 'directivas' se caracterizan por un discurso autoritario y prescriptivo; el educador dirige el proceso de intervención mientras que el profesor-estudiante ejecuta. En las intervenciones «alternativas» el educador plantea al profesor-estudiante una serie de alternativas para que éste pueda realizar la elección más apropiada. Por último, en la relación «no directiva» el educador usa un discurso propio de un profesional experto, comprensivo, reflexivo, y flexible para facilitar el proceso de exploración y desarrollo profesional del estudiante.

Randall y Thorton (2001) destacan dos enfoques de intervención: autoritario y facilitador. En el primero, el consejero indica al profesor-estudiante lo que tiene que hacer y cómo mejorar o modificar la manera de enseñar. En el segundo, el profesor-estudiante expresa sus emociones y sentimientos y el consejero promueve la autoexploración a través del cuestionamiento sobre puntos críticos y la valoración de lo realizado (Orland-Barak y Klein, 2005).

\subsection{La retroalimentación efectiva}

Randall y Thornton $(2001,13)$ señalan que «un método popular para dar retroalimentación sobre la enseñanza, a nivel de formación inicial docente, es la mentoría». No obstante, no todas las interacciones resultan ser una ayuda efectiva pues ser mentor requiere no tan sólo de experiencia profesional en la especialidad sino también contar con habilidades personales y sociales que le otorgan credibilidad en el rol de formación. 
Al respecto, Malderez y Wedell (2007, 88-89) opinan: «las dos funciones principales del mentor son escuchar y conversar de manera bien informada y estructurada... la mentoría más que 'entregar' algo, consiste en ayudar al aprendiz a descubrir y hacer las conexiones por ellos mismos».

\subsection{El proceso de comunicación en la mentoría}

Tobón (2008) señala que comunicar significa compartir y establecer una cierta participación en común con alguien. Los tres elementos necesarios para que se produzca esta comunicación son: (a) una fuente, (b) un mensaje y (c) un destino.

La comunicación es un proceso en el cual la fuente y el destino están sintonizados - no-lineal — cada persona es fuente y destino a la vez. Asimismo, en la comunicación humana se utiliza una pluralidad de canales: la expresión del rostro, la gestualidad, la postura del cuerpo y la relación espacial.

En la comunicación, el emisor transmite un mensaje al destinatario, teniendo ambos un código común, mediante el cual el emisor codifica y el destinatario decodifica el mensaje. El último elemento es el contacto, es decir, el canal físico y la conexión psicológica entre ambos.

\subsection{Exploración de las creencias en los procesos de mentoría}

Prieto (2008) y Díaz, Martínez, Roa y Sanhueza (2010) señalan que para tratar de comprender cómo los profesores se relacionan con la enseñanza, es necesario examinar las creencias, los procesos que subyacen a las acciones de los profesores en la sala de clases.

Al respecto, Crookes (2003) señala que la comprensión que tiene uno mismo de su enseñanza es modelada tanto por el proceso de formación docente que se ha recibido como por todas las experiencias que modelan la vida y hacen la persona y el profesor que uno es. No obstante, destaca que los profesores a menudo encuentran difícil articular las creencias y actitudes que subyacen en la manera que ellos enseñan; por eso es importante examinar esas creencias como un primer paso en decidir lo que a uno le gustaría cambiar y cómo. El proceso de exploración de las creencias es esencial en el entrenamiento de los profesores mentores. 


\subsection{Pensamiento reflexivo}

Zabala y Arnau (2009) y Markee (2009) destacan la práctica reflexiva desde dos enfoques: reflexión-sobre-acción y reflexión-en-acción. En el contexto educacional, la primera corresponde a la reflexión que ocurre antes y después de una clase. La segunda se refiere a la reflexión que puede darse durante el transcurso de la acción. Los profesionales que combinan ambos tipos de reflexión, participan de un proceso de aprendizaje auto-dirigido basándose en la experiencia personal.

Cabe mencionar que los enfoques recientes destacan que en las interacciones de mentoría, el proceso reflexivo comienza con la descripción de algo visible, para luego ir más allá de la acción y dar paso al ciclo de la revisión, selección, aprendizaje y plan de acción, a través de "preguntas reflexivas» formuladas por el mentor (Malderez y Wedell, 2007; Orland-Barak y Klein, 2005).

\subsection{Construcción de nuevos conocimientos}

Otra mirada alternativa al proceso de aprendizaje que experimentan los profesores-estudiantes, es el propuesto por los constructivistas sociales, quienes en el contexto de la práctica docente, señalan que mediante la provisión de desafíos focalizados y preguntas por parte del mentor, el profesor-estudiante internaliza las ideas nuevas - proceso de andamiaje (Orland-Barak, 2002). A través de este proceso, éste adopta y adapta los conceptos para así construir su propia versión- apropiación. El rol del mentor consiste en identificar la Zona de Desarrollo Próximo del aprendiz y proveer andamiaje para ayudarlo a avanzar e internalizar ideas, conceptos y habilidades nuevas.

\section{DISEÑO DE LA INVESTIGACIÓN}

\subsection{Objetivo general}

Comprender los patrones de comunicación que se establecen en la relación entre el mentor y el profesor-estudiante de la carrera de Pedagogía en Inglés de una institución superior chilena.

\subsection{Tipo de estudio}

La presente investigación es un estudio de caso múltiple que se adscribe al paradigma interpretativo-hermeneútico de tipo cualitativo. Esta de- 
finición permitió la exploración, descripción, análisis e interpretación de los hallazgos mediante el contraste de la información obtenida en el grupo de mentores y los profesores-estudiantes.

\subsection{Técnica de recolección de datos}

La técnica de recolección de información utilizada es la entrevista semiestructurada (ver extractos de las entrevistas en el anexo). Según Creswell (2003) la entrevista es uno de los medios para acceder al conocimiento, las creencias, los rituales, la vida de esa sociedad o cultura, obteniendo datos en el propio lenguaje de los sujetos. Se desarrolla a partir de preguntas que persiguen reconstruir lo que para el entrevistado significa el problema objeto de estudio. De esta manera, mediante la aplicación de una entrevista semi-estructurada, la presente investigación intentó capturar las interpretaciones de cada uno de los sujetos informantes respecto de dos ideas centrales: la conceptualización de mentoría y los patrones de comunicación que se establecen en las relaciones de mentoría.

\subsection{Informantes}

Los informantes de este estudio son seis profesores de inglés con perfeccionamiento en mentoría, otorgado en una institución de educación superior de la octava región, Chile a través del programa piloto de Mentores del MINEDUC del año 2005. Todos han participado activamente en el área de práctica pedagógica de una carrera de Pedagogía en Inglés, mediante la implementación de procesos de mentoría con estudiantes en práctica. Asimismo, los seis profesores imparten clases de inglés en establecimientos educacionales de la región; cuatro de ellos trabajan en el sistema público, una en un colegio semi-público, y una en un colegio privado. Se suman a los profesores, seis estudiantes de la carrera de Pedagogía en Inglés que realizan su práctica profesional final en establecimientos educacionales de la octava región en Chile. En suma, los informantes son parte de una muestra no probabilística intencionada de seis profesores de inglés capacitados como profesores mentores y seis profesores-estudiantes que realizan su práctica profesional final.

\subsection{Procedimiento de análisis de los datos}

La riqueza de la información cualitativa, que se capturó mediante la aplicación de la entrevista semi-estructurada, se operacionalizó mediante la técnica cualitativa de análisis de contenido semántico estructurado. Al 
respecto, Flick (2004) y Cisternas (2005) indican que el análisis de contenido es uno de los procedimientos clásicos para analizar el material textual, con independencia de la procedencia de éste. Esta técnica permitió comprender en profundidad los patrones de comunicación que se establecen al interior del proceso de mentoría. Posterior a la aplicación de la entrevista semi-estructurada a los doce sujetos informantes, se procedió a realizar las siguientes tareas: (1) vaciar la información relevante seleccionada en una hoja de codificación diseñada según la estructura y las temáticas pre-establecidas de la entrevista y (2) organizar la información recopilada en la hoja de codificación, según los criterios de relevancia, frecuencia y pertinencia de las categorías y subcategorías establecidas en congruencia con el marco teórico y el objeto de estudio. Se estableció una codificación abierta, que consistió en el examen y fragmentación de la información recopilada para organizarla en dimensiones y categorías. La codificación inicial de esta etapa se realizó con el apoyo del software Atlasti, que facilitó la tarea de almacenamiento, sistematización y codificación de los textos. En general, la etapa de codificación abierta siguió el principio de comparación constante.

Se consideró, además, la triangulación de los datos por informante y por investigador durante la investigación, con el propósito de levantar las categorías pre-establecidas y emergentes, proceso que permitió asegurar la confiabilidad de dicho análisis. Asimismo, se consideró la técnica de saturación especialmente en la triangulación de los datos pues permitió validar el proceso de levantamiento de las categorías como resultado de la repetición y contraste sistemático de la información, sin obtener nuevas interpretaciones.

\section{ANÁLISIS Y DISCUSIÓN DE LOS RESULTADOS}

\subsection{Análisis de las entrevistas de los profesores mentores y profesores-estudiantes}

Los datos arrojados por las entrevistas han sido organizados, a partir de la comparación de las respuestas entregadas tanto por los profesoresmentores como por los profesores-estudiantes, según la literatura revisada y los conceptos que emergieron del análisis de contenido semántico. 


\begin{tabular}{|c|c|}
\hline Dimensiones & Categorías \\
\hline $\begin{array}{l}\text { - Conceptualización y características } \\
\text { del proceso de mentoría }\end{array}$ & $\begin{array}{l}\text { - Concepto de mentoría } \\
\text { - Beneficios del proceso de mentoría } \\
\text { - Los roles del profesor mentor y del } \\
\text { profesor-estudiante } \\
\text { - Problemáticas asociadas a la mentoría } \\
\text { - Impacto de la mentoría en la forma- } \\
\text { ción inicial docente }\end{array}$ \\
\hline $\begin{array}{l}\text { - Patrones de comunicación en el pro- } \\
\text { ceso de mentoría }\end{array}$ & $\begin{array}{l}\text { - Tipos de interacción entre el mentor y } \\
\text { el profesor-estudiante } \\
\text { - Elementos de la comunicación verbal y } \\
\text { no-verbal en el proceso de mentoría }\end{array}$ \\
\hline - Componente cognitivo en mentoría & $\begin{array}{l}\text { - Procesos cognitivos de mentores y pro- } \\
\text { fesores-estudiantes } \\
\text { - Desarrollo del pensamiento reflexivo } \\
\text { en mentores y profesores-estudiantes }\end{array}$ \\
\hline
\end{tabular}

Tabla 1. Organización de las dimensiones y categorías

\subsubsection{Primera dimensión: Conceptualización y características del proceso de mentoría}

\section{Categoría I: Concepto de mentoría}

Los profesores mentores definen mentoría como un proceso de guía y apoyo y de crecimiento mutuo y como una relación establecida entre quien sabe más (profesor-mentor) y quien está aprendiendo (profesor-estudiante). Según esto, el informante 2 declara: (mentoría es) una guía hacia la docencia, un apoyo para quien empieza dirigido a mejorar su práctica docente.

Los profesores-estudiantes, en cambio, definen la mentoría como un proceso de aprendizaje mutuo basado en una relación más cercana y horizontal en la que el profesor mentor ayuda, apoya y guía a través de sus conocimientos y experiencia. Según esto, el informante 6 declara:

...(mentoría) es...un proceso en el cual el profesor guía (mentor) se acerca más al alumno (profesor-estudiante)....aparte de una relación profesional...se crean lazos...uno puede aprender del profesor (y) el profesor igual de uno...

Categoría II: Beneficios del proceso de mentoría 
Respecto de los beneficios de la mentoría, el grupo de mentores destacan los siguientes puntos: La mentoría permite refrescar la experiencia de los mentores; promover la reflexión, el crecimiento y el aprendizaje mutuo; establecer un diálogo abierto; provocar un impacto en la formación inicial docente; y favorecer el desarrollo personal y profesional del mentor. Por ejemplo, el informante 5 afirma:

...deberían tener todos los estudiantes...esta oportunidad de poder explorarse a sí mismos...y (decir) si...elegí la pedagogía...porque estoy dispuesto a preocuparme por el desarrollo y crecimiento del...otro que es mi prójimo;....mentoría te rescata esos valores,...

Para los profesores-estudiantes existen un conjunto de ventajas que destacan en el proceso de mentoría, como por ejemplo: el lenguaje común entre el tutor universitario y el mentor; el alto nivel de involucramiento de ambos interlocutores; la cercanía, la confianza y la seguridad del proceso; el aprendizaje mediante la crítica constructiva; la exploración y cambio profesional; el fuerte impacto en su proceso de formación pedagógica y profesional; y, por último, el diálogo reflexivo. Al respecto, el informante 6 señala:

...yo creo que me sirvió mucho...el hecho de recibir críticas constructivas (de) cosas...que para uno son detalles pero que son súper importantes...me sirvió mucho...para saber qué...(y) cómo puedo mejorar a futuro.

Categoría III: Los roles del profesor mentor y del profesor-estudiante

En relación con los roles y funciones del profesor mentor, se declaran los siguientes: guía, apoyo y acompañantes en el proceso de formación de los profesores-estudiantes, aculturador, explorador, observador y consejero. Al respecto, el informante 2 afirma:

Mi rol...va más centrado hacia el apoyo...mi estilo es recoger las ideas del alumno en práctica y a partir de sus ideas, sus conceptos, empezar a guiar, a trabajar, a encauzar en términos prácticos después estas ideas.

Por otra parte, los mentores declaran que el profesor guía en comparación con el mentor, cumple roles y funciones muy distintos, puesto que el primero cumple sólo un rol «evaluador», mientras que el segundo guía y apoya el desarrollo profesional y personal del profesor-estudiante. El informante 4 señala:

...antes uno veía las cosas negativas de la otra persona y de corregirles esto y lo otro...; ellos mismos (profesores-estudiantes) expresan que hay una diferencia...cuando son guiados y apoyados por un profesor mentor a un profesor guía. 
Los profesores-estudiantes, por su parte, señalan que su rol y función es pro-activo, colaborativo, responsable y comprometido, lo que demuestra ser comparativamente distinto al rol pasivo que desarrollan en un programa de práctica inicial tradicional. De esta manera, ellos declaran que el profesor mentor los ayuda en su proceso de aprendizaje, pues promueve la reflexión y profundización de los temas, a la vez de conocer y apoyar las tareas y trabajos de observación que les son requeridos en la universidad. Al respecto, el informante 1 afirma:

(en mentoría) fue un rol netamente activo, o sea, trabajamos a la par con la profesora (mentora).

Categoría IV: Problemáticas asociadas a la mentoría

Entre las problemáticas, los profesores mentores mencionan: el tiempo de dedicación a este proceso, la no institucionalización de la mentoría a nivel nacional, regional y local; y la descoordinación entre el mentor y el tutor de la institución superior. Al respecto, el informante 2 declara:

...como estos planes de mentoría no están oficializados en los colegios, el profesor a cargo de un alumno (profesor-estudiante) o de un grupo (de éstos), no tiene destinado un tiempo de trabajo para ello...yo encuentro que es necesario.

Los profesores-estudiantes, por su lado, identifican las siguientes problemáticas: la falta de mayor tiempo de permanencia en los establecimientos educacionales, un programa no institucionalizado en las universidades y el tiempo insuficiente para el ensayo y error en su desempeño pedagógico. Según esto, el informante 5 señala:

...a lo mejor (la mentoría) consume más tiempo de lo que yo...usaba para mis prácticas anteriores...se necesita disponer de una hora para ir al colegio, más allá de la que uno va el día de las prácticas.

Categoría V: Impacto de la mentoría en la formación inicial docente

Los seis profesores-mentores destacan el rol de la mentoría en la formación inicial docente, puesto que se evidencia un impacto positivo en los profesores estudiantes debido al apoyo permanente del mentor en su formación profesional, lo que se ilustra en este fragmento:

Informante 1: (mentoría) de todas maneras tiene un impacto...el futuro profesor llega a un lugar...con un apoyo (del mentor);...(pues) no es lo mismo...empezar a buscar,...investigar, a que...le vayan mostrando las posibili- 
dades que se pueden dar..., en el sentido que puede pasar esto (o) lo otro y (el profesor-estudiante) opta.

La formación inicial de los profesores-estudiantes se beneficia directamente con los procesos de mentoría pues estos últimos promueven el mejoramiento de las prácticas pedagógicas, el pensamiento pedagógico y una reafirmación vocacional a través de la reflexión que sólo la mentoría otorga, lo que se enuncia a continuación:

Informante 5: ...deberían tener todos...esta oportunidad de poder explorarse a sí mismos...y (decir) si...elegí la pedagogía...porque estoy dispuesto a preocuparme por el desarrollo y crecimiento del...otro que es mi prójimo;...mentoría te rescata esos valores,....esta sociedad...necesita profesores a través de la reflexión.

Se hace cada vez más necesario dentro de los programas de formación inicial docente contar con programas de mentoría, pues esto permite acortar los procesos de aprendizaje de los profesores noveles en su período de inserción laboral, lo que se ilustra en el siguiente fragmento:

Informante 2: Las competencias que deben tener (los profesores recién egresados) no son solamente saber la lengua y...algunas técnicas de enseñanza..., requieren más competencias; entonces el contacto con una persona que tiene experiencia, los hace enriquecerse más y acortar este período de aprendizaje que significa empezar a trabajar.

En el caso de los profesores-estudiantes, los seis informantes opinan que la relación establecida con sus profesores mentores tuvo una influencia positiva en su proceso de formación profesional y pedagógica. Esto se observa en el siguiente fragmento:

Informante 1: De todas maneras (hay impacto en la formación inicial docente) porque....ahora que estoy con una profesora mentora...claramente...fui superándome a mí misma)...

Además el aprendizaje logrado en dicho proceso es mayor por tratarse de una enseñanza holística de la educación por parte del profesor mentor. Esto se declara en el fragmento a continuación:

Informante 5: Sí, yo creo que sí (muy segura) (hay impacto en la formación inicial docente), se aprende el doble, el triple de lo de una práctica sin profesor mentor porque (éste)...enseña en todos los ámbitos que envuelve la educación. 


\subsubsection{Segunda dimensión: Patrones de comunicación en el proceso de mentoría} diante

Categoría I: Tipos de interacción entre el mentor y el profesor-estu-

Según el grupo de profesores-mentores, los tipos de interacción al interior del proceso de mentoría se caracterizan por ser más bien dialógicos y/o no-directivos con el propósito de promover el desarrollo, tanto profesional como personal, de los profesores-estudiantes. El profesor mentor fomenta un diálogo con el profesor-estudiante, a partir de las experiencias pedagógicas comunes que permitan a este último, desarrollar el pensamiento crítico-reflexivo y descubrir sus propias creencias y preconcepciones a través de un proceso de exploración. Cabe señalar que estas interacciones necesitan de ciertas habilidades sociales y comunicativas del profesor-estudiante, tales como: ser respetuoso, tolerante y estar dispuesto a escuchar y dialogar. Asimismo, estas interacciones requieren de un profesor-mentor capaz de ser creíble para el profesor-estudiante. Al respecto, el informante 3 señala:

... es más bien (una intervención) dialógica...nunca una prescriptiva porque creo que las recetas no pueden existir...es dialógico...un crecimiento mutuo en ambos sentidos.

Según los profesores-estudiantes, las conversaciones de mentoría se caracterizan por ser más bien dialógicas y/o facilitadoras y promueven el apoyo y la reflexión mutua. No obstante, algunas interacciones pueden ser de tipo más directivo dependiendo del contenido de la conversación. Asimismo, éstos señalan que las experiencias personales y pedagógicas comunes han sido la base de la discusión y el aprendizaje efectivo. Por otra parte, ellos opinan que para potenciar el diálogo fluido y enriquecedor, el profesor mentor debe tener habilidades sociales y comunicativas específicas. Al respecto, el informante 1 afirma:

...la profesora...me sugería cosas (y) yo también a su vez le sugería...el hecho de que ella me diera sugerencias y corrigiera...fue de gran ayuda,...realmente fue de apoyo mutuo .

Categoría II: Elementos de la comunicación verbal y no-verbal en el proceso de mentoría

Con respecto a la comunicación verbal, los profesores mentores destacan: el tono y entonación de la voz, las pausas, y el parafraseo como elementos importantes al momento de interactuar con los profesores-estu- 
diantes. Cabe mencionar que la mayoría de ellos opinan que las repeticiones de discurso son irrelevantes pues no tienen un aporte significativo en la interacción de mentoría. Por ejemplo, el informante 3 afirma:

... el tono puede demostrar ansiedad, enojo, frustración;....si no tengo un tono de voz adecuado tampoco me van a querer escuchar,...(ni) entender bien mi mensaje que estoy tratando de decodificar.

En el caso de los elementos de la comunicación no-verbal, la mayoría de los mentores señalan que éstos tienen una contribución importante en las interacciones de mentoría, entre los que destacan la gestualidad y la postura. En el caso de la gestualidad, opinan que gestos como: la sonrisa, el contacto visual y el movimiento de cejas y manos, contribuyen a crear un ambiente de fluidez, naturalidad, confianza y apoyo. En relación con la postura, ellos piensan que ésta contribuye a comunicar con el cuerpo la idea de acoger y dar confianza al profesor-estudiante de manera de lograr una comunicación efectiva y enriquecedora. Según esto, el informante 4 señala:

(los elementos de la comunicación no-verbal) contribuyen a que este diálogo (de mentoría) sea más natural (y) con más acogida...

Por su parte, en la comunicación verbal, los profesores-estudiantes destacan el tono y la entonación de la voz y el parafraseo, puesto que les asegura saber y comprender las temáticas involucradas en el diálogo. En el caso de las repeticiones, la mayoría de ellos opina que éstas contribuyen a enfatizar ideas y facilitar el parafraseo. Según esto, el informante 2 declara:

El número uno yo creo que sería...el tono y volumen de voz...porque...mi profesora mentora siempre tenía un tono muy amable (y) maternal...para mí eso...era muy importante.

Todos los profesores-estudiantes consideran que los elementos no verbales son muy relevantes en las conversaciones de mentoría, ya que contribuyen a fortalecer la comunicación y reafirmar los mensajes. Entre los que se destacan, están la gestualidad y la postura, pues demuestran el interés de los profesores mentores en la interacción con ellos y el nivel de acuerdo y aprobación de los mismos; además, ayudan a crear la confianza que se necesita para establecer una comunicación fluida. En el caso de la postura, la mayoría señala que ésta contribuye a crear una relación más cercana con sus profesores mentores, basada en la confianza. Al respecto, el informante 2 afirma: 
...yo creo que en todo sentido la gestualidad es importante porque se supone que uno...está buscando confianza en su profesora mentora.

\subsubsection{Tercera dimensión: El componente cognitivo en el proceso de mentoría}

Categoría I: Procesos cognitivos de mentores y profesores-estudiantes

Los mentores opinan que el saber teórico y la exploración explícita de las creencias, preconcepciones y prejuicios del profesor-estudiante tributan a un cambio y mejoramiento de sus prácticas pedagógicas, basado en la reflexión mutua; en otras palabras, destacan que las interacciones de mentoría estimulan el desarrollo del pensamiento crítico-reflexivo, la aceptación de la crítica constructiva y la toma de conciencia de la acción pedagógica de ambos. Según esto, el informante 2 afirma:

Yo creo que la principal (contribución de mentoría) es el hecho de que tengan a alguien....al lado observando..., preguntando (mentor)...pretende...mejorar los procesos acortando los tiempos de aprendizaje como futuros profesionales.

Los profesores-estudiantes consideran que las interacciones de mentoría promueven el desarrollo del pensamiento crítico y reflexivo permanente de sus prácticas pedagógicas y sus concepciones, y el aprendizaje y crecimiento profesional mutuo. Agregan que las interacciones con sus profesores mentores contribuyen a un aprendizaje significativo basado en un diálogo activo, participativo, reflexivo y exploratorio, destacando la competencia pedagógica y profesional de sus mentores. Por último, algunos señalan que la mentoría permite una mayor conexión entre el conocimiento teórico y el conocimiento práctico, puesto que el saber teórico-práctico de sus profesores mentores facilita que se relacionen y adecuen ambos saberes. Al respecto, el informante 5 señala:

Llevar lo teórico a la práctica ha sido...lo más importante porque ella (mentora) es la que ha trabajado con los alumnos...conoce bien cómo van a reaccionar frente a las actividades, entonces eso ha servido harto.

Categoría II: Desarrollo del pensamiento reflexivo en mentores y profesores-estudiantes

Para los mentores, la experiencia de mentoría les ha permitido desarrollar la reflexión y autocrítica con respecto a sus propias prácticas pedagógicas y les ha posibilitado estar más consciente de su acción pedagó- 
gica cotidiana, con el propósito de mejorarla y reflexionar sobre la misma en conjunto con el profesor-estudiante. Esto se ilustra en el siguiente fragmento:

Informante 3:...a medida que uno se va involucrando más en el proceso de mentoría, uno empieza a reflexionar sobre sus prácticas...está consciente del actuar pedagógico para poder conversar o reflexionar sobre eso...

Asimismo, las conversaciones abiertas y respetuosas que se establecen entre el mentor y el profesor-estudiante en mentoría, promueven una relación dialógica capaz de aceptar la crítica constructiva de ambos. Esto se enuncia en el fragmento a continuación:

Informante 5: la conversación abierta (y) respetuosa te promueve un diálogo cada vez...más serio y delicado,...y aprovecha uno (mentor) para orientar y guiar...y llevarlos a que ellos (profesores-estudiantes) acepten la crítica...

Los profesores-estudiantes, por su parte, destacan que la mentoría promueve la reflexión no sólo de aspectos técnicos propios de la enseñanza del idioma sino que, además, de diversos temas sobre educación, lo que amplía su visión del proceso de prácticas pedagógicas, como se evidencia en el siguiente fragmento:

Informante 3: ...en este grupo de mentoría se reflexiona un poco más sobre educación (y) de las prácticas y todo eso me ayuda a tener...una visión más amplia quizás de todo esto y también de las prácticas pedagógicas.

Igualmente, la explicitación que realiza el profesor-mentor acerca de las fortalezas y aspectos por mejorar en las conversaciones, promueve un proceso de reflexión y exploración permanente de los estudiantes sobre su desempeño docente. Esto se declara en el siguiente fragmento:

Informante 2: Sí...(las interacciones) ayudan...(al desarrollo) del pensamiento crítico porque ella me decía las cosas que yo podía mejorar, pero no lo que tenía que hacer;....al darme esas...críticas constructivas...me hacía pensar en lo que podía mejorar y...agregar a mi comportamiento como futura profesora.

Además, las interacciones de mentoría contribuyen a contraponer los conocimientos y opiniones de los estudiantes con los de su profesora mentora con respecto a una temática o situación dada, lo que resulta en un proceso de análisis e interpretación que incluye ambas visiones. Esto resulta crucial al momento de preparar y planificar un proyecto de intervención de aula, pues se realiza una conversación previa a la ejecución para evaluar 
en conjunto la clase planificada, lo que tributa al desarrollo del pensamiento crítico y reflexivo, como se declara en el fragmento que sigue:

Informante 5: ...cuando llega el momento de...realizar nuestra clase...y empezamos a evaluar...contenido,.... la planificación...se hace una evaluación general de...todo y...es donde....se da la comunicación de (lo) que puedes hacer...ahí se va reflexionando...

Por último, el planteamiento de preguntas respecto de situaciones de aula por parte de la profesora mentora contribuye a un proceso de reflexión permanente del profesor-estudiante. En síntesis, los análisis presentados en esta sección, pueden ser visualizados gráficamente en la figura 1 que sintetiza los datos arrojados por las dos entrevistas semi-estructuradas. 


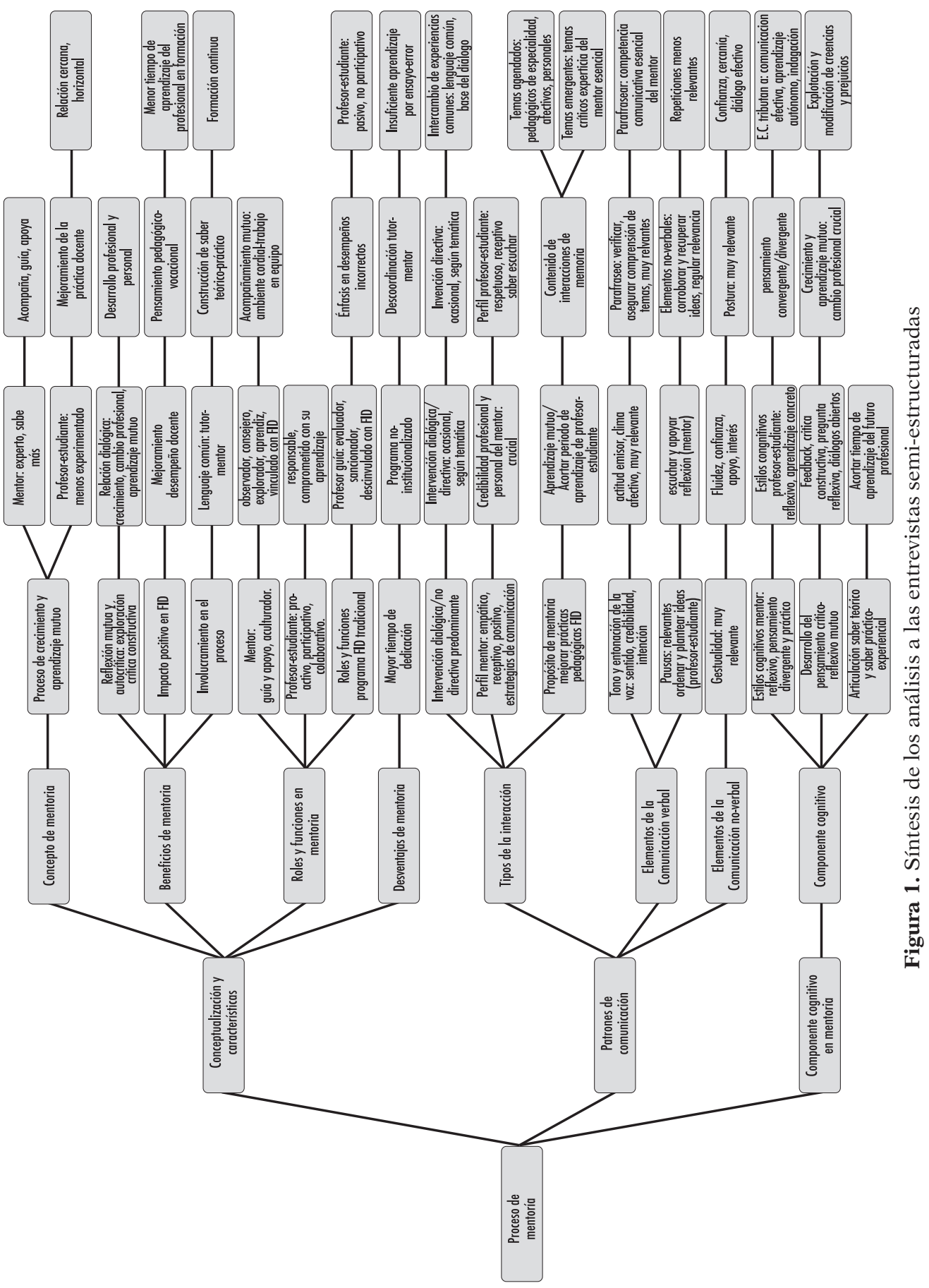




\section{MODO DE DISCUSIÓN Y CONCLUSIÓN}

Mentoría es definido como un proceso de crecimiento y aprendizaje mutuo basado en una relación cercana y horizontal entre un profesor mentor, experto y más experimentado, y un profesor-estudiante, en proceso de formación docente y menos experimentado. Asimismo, se caracteriza por establecer una relación dialógica entre ambos, lo que promueve una reflexión mutua y autocrítica, a través de la exploración de sus creencias, preconcepciones y prejuicios - especialmente del profesional en formación-y de la crítica constructiva.

Estos patrones de comunicación se caracterizan por tener un impacto significativo en la formación inicial docente, lo que tributa a acortar los tiempos de aprendizaje del profesional en formación. Además, se distingue el nivel de involucramiento del mentor y del profesor-estudiante y la construcción del saber teórico-práctico como parte del crecimiento y aprendizaje mutuo.

Igualmente, se determinan tres tipos de intervenciones del mentor al interior de las interacciones, a saber: prescriptiva/directiva; dialógica/prescriptiva; y dialógica/no directiva, destacándose estas últimas pues favorecen el pensamiento crítico-reflexivo. Otro patrón importante constituye el intercambio de las experiencias de campo de los participantes, pues son el lenguaje común y la base temática para comenzar a dialogar y sintonizar.

No obstante, estos patrones de comunicación requieren la adquisición de ciertas habilidades sociales y comunicativas del mentor y del profesor-estudiante que favorezcan la relación establecida entre ambos. Para el perfil del mentor se destacan: ser empático, receptivo a sugerencias, con una actitud positiva y un buen trato, creíble y que disponga de estrategias comunicativas efectivas. El perfil del profesor-estudiante, por su parte, implica: ser respetuoso, tolerante a las opiniones divergentes, receptivo a sugerencias y tener disposición a escuchar.

Por otra parte, los elementos de la comunicación verbal y no-verbal corresponden a patrones de comunicación altamente valorados por los informantes. En los elementos de la comunicación verbal se definen: tono y entonación de la voz, parafraseo y pausas; los que promueven el proceso de reflexión de los profesores estudiantes. Entre los elementos de la comunicación no-verbal destacan la gestualidad y postura pues tributan a crear un ambiente de confianza, apoyo y cercanía.

Asimismo, los informantes destacan el componente cognitivo como un patrón de comunicación relevante en mentoría pues promueve: el pensa- 
miento crítico-reflexivo, el crecimiento y aprendizaje mutuo, la exploración y modificación de las creencias y prejuicios, y la articulación del saber práctico- experiencial del mentor con el saber-teórico del profesor-estudiante.

Por otra parte, los mentores definen como sus principales roles y funciones ser guía y apoyo, aculturador, observador, consejero, explorador, aprendiz y vinculado con los lineamientos de la formación inicial docente. Entre los roles y funciones de los profesores-estudiantes se destacan ser pro-activo, participativo, responsable y comprometido con su proceso de aprendizaje.

Finalmente, con la formación de una tríada formativa entre el tutor universitario, el mentor y el profesor-estudiante al interior de la relación de mentoría se puede contribuir a optimizar la vinculación entre las instituciones superiores formativas con los establecimientos educativos de la región y del país. Además, la identificación de las problemáticas asociadas al proceso de mentoría contenidas en la presente investigación permite entregar una retroalimentación oportuna y efectiva al grupo de mentores participantes para un posible plan de acción y, a la vez, retroalimentar a otras experiencias de mentoría que actualmente estén siendo implementadas a nivel nacional e internacional. Por lo tanto, los resultados de este estudio podrían constituirse en una "VoZ» con una potencialidad intrínseca para orientar, guiar y apoyar la implementación de futuros programas de mentoría en los programas de formación de profesores.

\section{NOTAS}

1. Esta artículo se inserta en el contexto del Proyecto gubernamental FONDECYT N ${ }^{\circ}$ 1085313/2008 «El Sistema de Cognición Docente, las Actuaciones Pedagógicas del Profesor de Inglés Universitario y su Impacto en la Enseñanza-Aprendizaje del Idioma».

2. El Programa «Inglés Abre Puertas» es un plan de 10 años de duración que tiene por objetivo mejorar la calidad de la enseñanza y aprendizaje del inglés en el sistema escolar chileno. 


\section{REFERENCIAS BIBLIOGRÁFICAS}

Ávalos, B. (2002). Profesores para Chile, Historia de un Proyecto. Santiago de Chile: Ministerio de Educación.

Cisternas, F. (2005). Categorización y triangulación como procesos de validación del conocimiento en investigación cualitativa. Theoria. 14 (1), 61-71.

Creswell, J. (2003). Research design. Qualitative, quantitative and mixed methods and approaches. Thousand Oaks, CA: Sage Publications.

Crookes, G. (2003). A practicum in Tesol. Professional development through teaching practice. Cambridge: Cambridge Language Education.

Díaz, C.; Martínez, P.; Roa, I.; Sanhueza; M. (2010). Los docentes en la sociedad actual: sus creencias y cogniciones pedagógicas respecto al proceso didáctico. Polis, 9 (25), 421-436.

Feiman-Nemser, S. (2003). What new teachers need to learn. Educational Leadership: Keeping good teachers. 60 (8), 25-29.

Flick, U. (2004). Introducción a la investigación cualitativa. Madrid: Ediciones Morata, S.L.

Malderez, A.; Wedell, M. (2007). Teaching teachers: Processes and practices. Londres: Continuum International Publishing Group.

Markee, N. (2009). Managing curricular innovation: Cambridge: Cambridge University Press.

Nation, I.S.P.; Macalister, J. (2010). Language curriculum design. London: Routledge.

Orland-Barak, L. (2002). The impact of the assessment of practice teaching on beginning teaching: Learning to ask different questions. Teacher Education Quaterly, 12 (3), 67-78.

Orland-Barak, L. (2003). Emergency room stories: mentors at the intersection between the moral and the pedagogical. Journal of In-service Education, 29, (3), 489-512.

Orland-Barak, L.; Klein, S. (2005). The expressed and the realized: Mentors' representations of a mentoring conversation and its realization in practice. Teaching and Teacher Education, 21, (4), 379-402.

Orland-Barak, L. (2005). Lost in translation. Mentors learning to participate in competing discourses of practice. Journal of Teacher Education, 56, (4), 355-366.

Pimienta, J. (2008). Constructivismo. Estrategias para aprender a aprender. Ciudad de México: Pearson.

Prieto, L. (2008). La enseñanza universitaria centrada en el aprendizaje. Barcelona: Octaedro.

Randall, M.; Thornton, B. (2001). Advising \& supporting teachers. Cambridge: Cambridge University Press.

Sotomayor, C.; Walker, H. (2009). Formación continua de profesores. ¿Cómo desarrollar competencias para el trabajo escolar? Santiago de Chile: Editorial Universitaria.

Tobón, S. (2008). Formación basada en competencias. Pensamiento complejo, diseño curricular y didáctica. Bogotá: Ecoe.

Zabala, A. y Arnau, L. (2009). 11 ideas clave cómo aprender y enseñar competencias. Barcelona: Grao. 


\section{ANEXOS}

1. Extracto de la entrevista semiestructurada aplicada a los profesoresmentores

I. Preguntas generales sobre el proceso de mentoría

1. ¿Cómo definirías el concepto de "Mentoría”?

2. Según la experiencia que has tenido como profesor(a) mentor, ¿cuál ha sido tu rol en la relación establecida con el estudiante en práctica?

3. ¿Cuáles han sido las funciones que has realizado en dicha relación?

4. ¿Qué aspectos positivos y/o negativos destacarías de la interacción entre el profesor mentor y estudiante en práctica?

5. En tu opinión, ¿crees que los procesos de mentoría pueden tener un impacto en la formación inicial de los futuros profesores de inglés?

2. Extracto de la entrevista semiestructurada aplicada a los profesores-estudiantes

I. Preguntas generales sobre el proceso de mentoría

1. ¿Cómo definirías el concepto de «Mentoría»?

2. Según la experiencia que has tenido este semestre como estudiante en práctica, ¿cuál ha sido tu "rol» en tu relación con el profesor mentor?

3. ¿Cuáles han sido las funciones que has realizado en esta relación?

4. ¿Qué aspectos positivos y/o negativos destacarías en el proceso de interacción entre el/la profesor mentor y el estudiante en práctica?

5. En tu opinión, ¿ crees que los procesos de mentoría pueden tener un impacto en tu formación inicial como futuro profesor de inglés? 


\section{PALABRAS CLAVE}

Mentoría, reflexión, interacción dialógica, rol del mentor, rol del profesor estudiante y crecimiento mutuo.

\section{KEY WORDS}

Mentoring, reflection, dialogic interaction, mentor's role, student teacher's role and mutual learning

\section{PERFIL ACADÉMICO Y PROFESIONAL DE LOS AUTORES}

Claudio Díaz Larenas es Doctor en Educación y Magíster en Lingüística por la Universidad de Concepción. Catedrático de Didáctica y Evaluación del inglés de la Universidad de Concepción. Investigador de la Dirección de Docencia. Sus principales líneas de investigación son: cognición docente, creencias pedagógicas y enseñanza y aprendizaje del inglés.

Cecilia Bastías Díaz es Magíster en Ciencias de la Educación por la Universidad Católica de la Santísima Concepción. Catedrática de Didáctica del inglés de la Universidad San Sebastián. Facultad de Ciencias de la Educación. Su principal línea de investigación es los procesos de mentoría.

Dirección de los autores: Claudio Díaz Larenas

Universidad de Concepción

Edmundo Larenas, 64 A.

Barrio Universitario.

Concepción, Chile

E-mail: claudiodiaz@udec.cl

Cecilia Bastías Díaz

Universidad San Sebastían

Sede Tres Pascualas

General Cruz 1577

Concepción, Chile

E-mail: cecilia.bastias@uss.cl

Fecha Recepción del Artículo: 08. Julio. 2010

Fecha Revisión del Artículo: 14. Octubre. 2010

Fecha Aceptación del Artículo: 16. Diciembre. 2010

Fecha de Revisión para publicación: 30. Junio. 2011 
